\title{
Accurate automatic tuning circuit for bipolar integrated filters
}

Wim J.A. de Heij ${ }^{1)}$, Klaas Hoen ${ }^{1)}$ and Evert Seevinck ${ }^{2)}$ (senior member IEEE)

1) University of Twente, Electrical Engineering Dept., IC-Technology and Electronics Group, P.O. Box 217, 7500 AE Enschede, The Netherlands.

\section{Abstract}

In this paper an accurate automatic tuning circuit, for tuning the cut-off frequency and Q-factor of high frequency bipolar filters, is presented. The circuit is based on a voltage controlled quadrature oscillator (VCO), is presented. The frequency and the RMS amplitude of the oscillator output signal are locked to the frequency and the RMS amplitude of a reference signal respectively. Special attention is paid to the actual Q-factor in the oscillator. Experimental results of a breadboard circuit operating from 136 to $317 \mathrm{kHz}$ are presented. Eventually the circuit will be scaled up in frequency and integrated for on-chip tuning of bipolar video frequency filters.

\section{Introduction}

Bipolar technology has been used before to realize integrated continuous-time filters at video frequencies, operating from 5V supply [1],[2]. Modern Bi-CMOS processing [3] allows the combination of analog bipolar filters with digital circuits on a single chip. Bipolar analog filters may become even more attractive for this combination if the supply voltage, being dictated by the CMOS digital circuits, goes down to $3.3 \mathrm{~V}$ in the future.

Recently a bipolar active-RC integrator (or transconductor-amplifier-C integrator) for video frequencies was presented [4]. In this integrator the high transconductance of the bipolar transistor was used effectively in the amplifier design. Furthermore, a simple tunable transconductor with good high-frequency properties and good linearity was designed for this integrator. Because of production tolerances and temperature dependence of integrated RC-products, automatic on-chip frequency tuning of filters is necessary to obtain the desired cutoff frequency. Especially at video frequencies we have to deal with a phase error (usually excess phase) of the integrator which must also be corrected by an automatic tuning circuit. Therefore the integrator circuit must have both tunable unity gain frequency and Q-factor.

\section{The integrator circuit}

Fig. 1 shows the concept of the balanced active-RC integrator [4]. To the left of the dashed line is a tunable transconductor, which is a parallel circuit of two fixed resistors $\mathrm{R}_{1+}=\mathrm{R}_{1-}=\mathrm{R}_{1}$ and a variable active transconductance $G_{V}$. To the right is a transconductance amplifier (TA) with transconductance $\mathrm{G}_{\mathrm{A}}$ with integration capacitors $\mathrm{C}_{1+}=\mathrm{C}_{1-}=\mathrm{C}_{1}$ and variable resistors $R_{\mathrm{Q}_{+}}=\mathrm{R}_{\mathrm{Q}_{-}}=\mathrm{R}_{\mathrm{Q}}$ in the feedback path. Output buffers drive the resistive load consisting of other transconductors. If the input and output resistance of the TA are assumed to be infinite, the following transfer function is found:

$\frac{v_{u}}{v_{i}}=\left(1+R_{1} G_{V}\right) * \frac{G_{A}^{*}\left(1-s C_{1}^{*}\left(-\frac{1}{G_{A}}-R_{Q}\right)\right)}{s C_{1}^{*}\left(1+G_{A} R_{1}\right)}$
2) Philips Research Laboratories, P.O. Box 80000 $5600 \mathrm{JA}$ Eindhoven, The Netherlands.

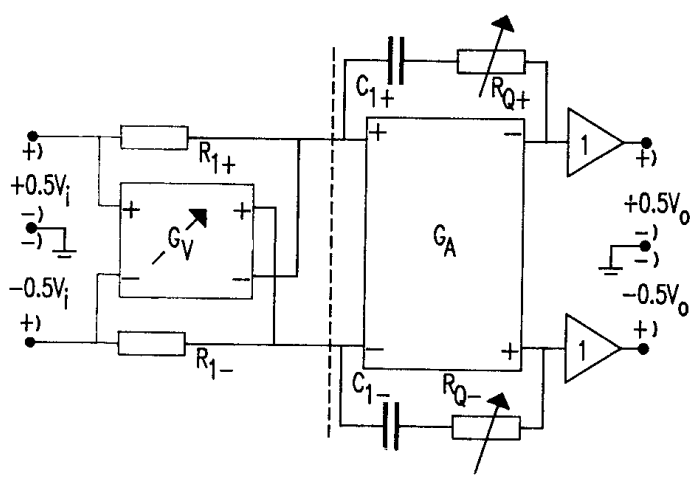

Fig. 1: Active-RC integrator concept.

The unity gain frequency $f_{o}$ is tuned by $G_{V}$ and phase is tuned by zero $s_{z}$, which is a function of the variable resistors $\mathrm{R}_{\mathrm{Q}}$ :

$$
\begin{aligned}
& \mathrm{f}_{\mathrm{o}} \approx \frac{1+\mathrm{G}_{\mathrm{V}} \mathrm{R}_{1}}{2 \pi \mathrm{R}_{1} \mathrm{C}_{1}} \quad\left(\text { if } \mathrm{G}_{\mathrm{A}} \mathrm{R}_{1}>>1\right) \\
& \mathrm{s}_{\mathrm{z}}=\frac{1}{\mathrm{C}_{1}{ }^{*}\left(\frac{1}{\mathrm{G}_{\mathrm{A}}}-\mathrm{R}_{\mathrm{Q}}\right)}
\end{aligned}
$$

The circuit realization of the tunable transconductor shown in fig. 2 has the following transconductance:

$$
\mathrm{G}=\frac{1}{\mathrm{R}_{1}}+\mathrm{G}_{\mathrm{V}}=\frac{1}{\mathrm{R}_{1}}+\frac{\mathrm{I}_{1}-\mathrm{I}_{2}}{\mathrm{I}_{3}} * \frac{1}{\mathrm{R}_{2}+\frac{1}{\mathrm{~g}_{\mathrm{m} 5,6}}}
$$

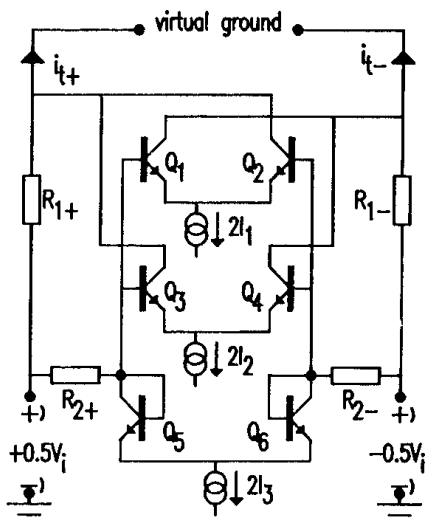

Fig. 2: Tunable transconductor circuit. 
The nominal design value is $1 / R_{1}$. The second term describes the active variable part, which is a function of $I_{1}-I_{2}$. With the difference of $I_{1}$ and $I_{2}$, which are both positive currents, the total transconductance $G$ can be tuned in both positive and negative direction.

Fig. 3 shows the circuit realization of the TA with feedback path and output buffers. The TA consists of differential pair $\left(\mathrm{Q}_{1}, \mathrm{Q}_{2}\right)$, biased with $2 \mathrm{I}_{\mathrm{S}}$. The diode connected transistors $\mathrm{Q}_{3}, \mathrm{O}_{4}$ act as the variable resistors $\mathrm{R}_{\mathrm{Q}_{+}}$and $\mathrm{R}_{\mathrm{Q}_{-}}$in the feedback path. While the collector current through $Q_{1}$ and $Q_{2}$ is fixed to $I_{S}$, the collector current through $\mathrm{Q}_{3}$ and $\mathrm{Q}_{4}$ is adjusted to $\mathrm{I}_{\mathrm{S}}-\mathrm{I}_{\mathrm{O}}$ by the CMFB circuit $\mathrm{Q}_{7}-\mathrm{Q}_{11}$ when the Q-tuning current $\mathrm{I}_{\mathrm{Q}}$ is injected into the circuit via current mirror $\mathrm{Q}_{12}-\mathrm{Q}_{14}$. If $\mathrm{I}_{\mathrm{Q}}>0$ then $\mathrm{R}_{\mathrm{Q}}=1 / \mathrm{g}_{\mathrm{n} 3,4}>1 / \mathrm{G}_{\mathrm{A}}=1 / \mathrm{g}_{\mathrm{m} 1,2}$ and the zero is in the left half plane. In this way excess phase can be compensated for. Emitter followers $\mathrm{Q}_{5}$ and $\mathrm{Q}_{6}$ are used as output buffers. More details are given in [4].

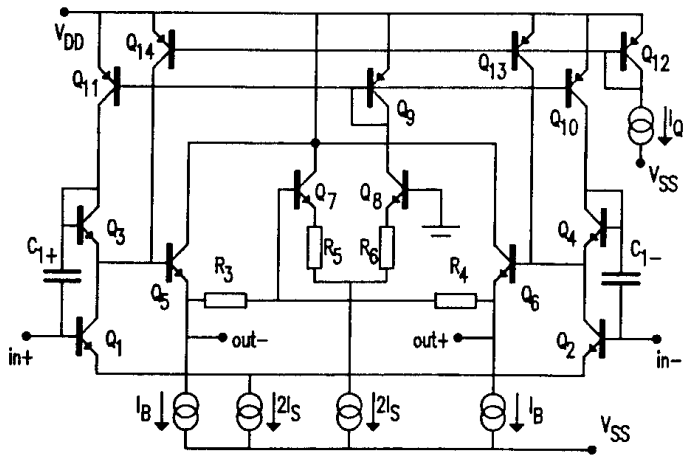

Fig. 3: Amplifier and feedback circuit.

\section{The tuning circuit}

Fig. 4 shows a block schematic of the complete tuning circuit. A quadrature oscillator, built as a two-integrator loop, is tuned in frequency and quality factor. From this tuning system control signals are derived which tune the cutoff frequency and O-factor of the slave filter [5].

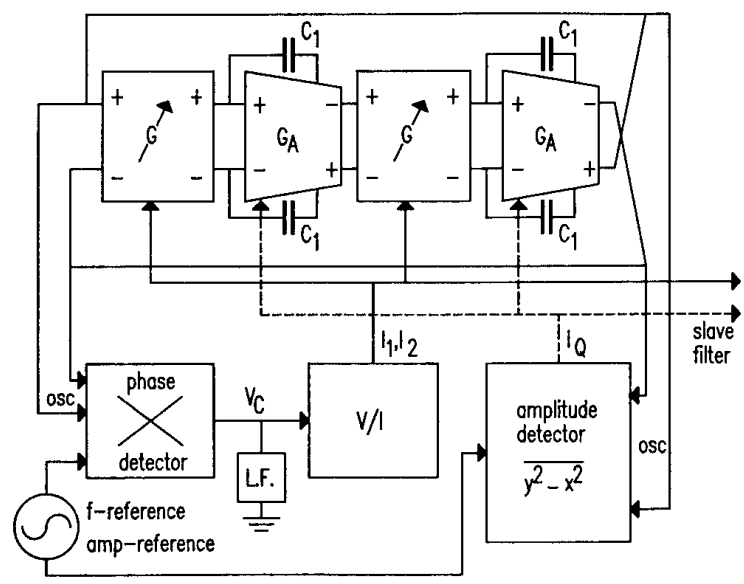

Fig. 4: Complete automatic tuning circuit.
The oscillation frequency $f_{\text {osc }}$ is locked to the external reference frequency by the PLL. The multiplier, used as a phase detector, has, a high output impedance resulting in a large voltage gain which makes a loop amplifier unnecessary. An emitter degenerated differential pair, used as $V / I$ converter, converts the control voltage $V_{C}$ into the tuning currents $I_{1}$ and $I_{2}$ which tune the variable transconductors. A passive $R C$ loop filter (L.F.) at the high impedance node optimizes PLL performance.

In the amplitude detector the difference of the squares of the oscillator output signal and the reference signal is averaged and amplified to produce the DC Q-tuning current $\mathrm{I}_{\mathrm{Q}}$, which tunes the phase of the integrators. If the oscillator output signal is too large the current $\mathrm{I}_{O}$ is increased, reducing the excess phase of the integrators and thus damping the oscillator. If the oscillator output signal is too small $\mathrm{I}_{Q}$ is decreased, increasing the excess phase of the integrators and thereby undamping the oscillator. The result will be that the RMS amplitude of the sine oscillator output signal is forced equal to the RMS amplitude of the reference signal, which may be a sine, square, triangle or other signal.

\section{Phase detector and amplitude detector}

Fig. 5 shows the Gilbert multiplier which is used as phase detector. The output currents are subtracted with an npn current mirror to avoid the $\beta$ error of a pnp mirror, which would result in a large offset, and to obtain an output voltage around $\left(V_{D D}+V_{S S}\right) / 2$, which is also the bias potential of both inputs.

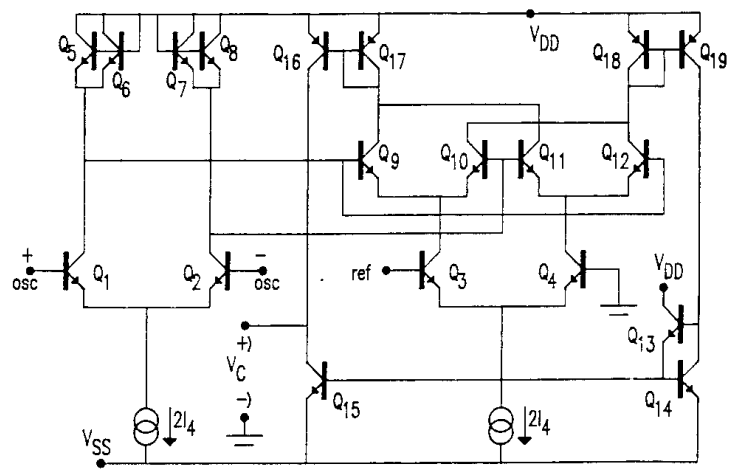

Fig. 5: Phase detector.

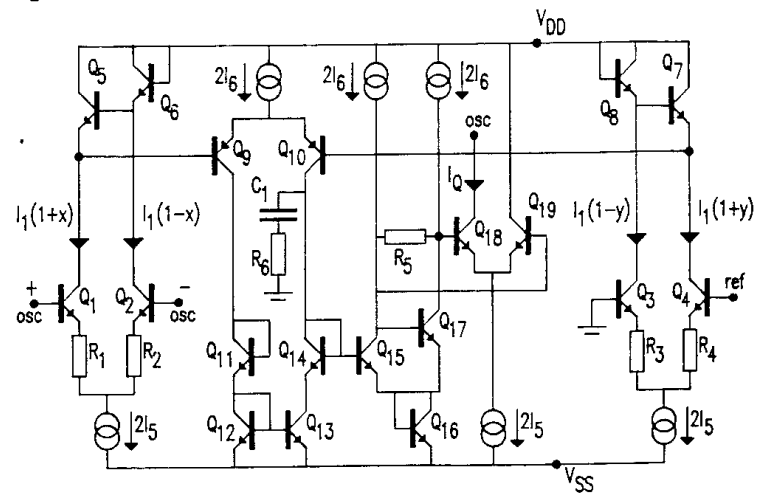

Fig. 6: Amplitude detector.

Fig. 6 shows the amplitude detector. The oscillator output signal and the reference signal are converted to currents $\mathrm{I}_{5}(1+\mathrm{x}), \mathrm{I}_{5}(1-\mathrm{x}) \quad$ and $\quad \mathrm{I}_{5}(1+\mathrm{y}), \mathrm{I}_{5}(1-\mathrm{y})$ respectively by identical V/I-converters $\left(\mathrm{Q}_{1}, \mathrm{Q}_{2}\right)$ and $\left(\mathrm{Q}_{3}, \mathrm{Q}_{4}\right)$. 
The translinear circuit $Q_{5}-Q_{10}$ gives an output current which is proportional with the difference of the squares of modulation index $x$ and $y$ :

$I_{\text {out }}=I_{c 10} I_{c 9}=2 I_{6} * \frac{x^{2}-y^{2}}{2-x^{2}-y^{2}}$

Output current $I_{\text {out }}$ is filtered by $C_{1}$. The $\mathrm{DC}$ component is amplified by the loop amplifier $Q_{15}-Q_{19}$ to give $I_{Q}$. For $\mathrm{DC}$ and low frequencies $\mathrm{Q}_{15}$ is current driven so that $\mathrm{I}_{\text {out }}$ is amplified by $\beta_{15} . \mathrm{Q}_{15}$ is biased at $2 \mathrm{I}_{6}$ to cancel the error $2 \mathrm{I}_{6} / \beta$ of mirror $\mathrm{Q}_{12}, \mathrm{Q}_{13}$ and thus reduce the offset. $Q_{17}$ acts as transimpedance amplifier with feedback resistor $R_{5}$. $I_{C 15}$ flows through $R_{5}$ and the voltage across $R_{5}$ is converted into $I_{Q}$ by $Q_{18}$ and $\mathrm{Q}_{19}$. The total current gain is equal to $\beta_{15} \mathrm{R}_{5} \mathrm{~g}_{m 18} / 2$, which was designed to be 350 . Because of the high loop gain, the average value of $\mathrm{I}_{\mathrm{out}}$ will be very small. It follows from (5) that the RMS-values of $x$ and $y$ will be equal, as required.

The current through $\mathrm{Q}_{16}$ is constant, so that the emitters of $\mathrm{Q}_{15}$ and $\mathrm{Q}_{17}$ are at virtual ground. The collector of $\mathrm{Q}_{10}$ is the only high impedance node in the amplitude detector, so that its frequency response is fully determined by the loop filter $\mathrm{R}_{6}, \mathrm{C}_{1}$.

\section{Start-up conditions and accuracy}

In the amplifier circuit shown in fig. 3 the current $I_{Q}$ can only be positive and therefore the zero $s_{z}$ can only be in the LHP or at infinity. As a result only excess phase can be compensated. If at $f_{\min }$ the excess phase is 0 for $I_{Q}=0$ the oscillator will only start up if reference frequency $f_{\text {ref }}>f_{\text {min }}$.

Besides $f_{r c f}>f_{\min }$ there is a second condition for correct start-up and convergence. If the oscillator does not oscillate, the control loops are in fact open. In such a situation the control signals $V_{C}$ and $I_{O}$ should take on values which tune the oscillator to a frequency $f_{\text {start }}>f_{\min }$ and undamp it. To achieve this the offsets in amplitude and phase detectors should be sufficiently small, which is the second condition.

Maximum Q-factor $\mathrm{Q}_{\mathrm{int}}$ of the integrators is achieved at low oscillation amplitude. At low amplitude the active elements in the oscillator operate in a linear mode, so that the pole positions are not signal dependent. In case of steady oscillation at such low amplitudes, the Q-tuning current $I_{O}$ must have a value which puts the poles exactly on the jw-axis and $I_{O}$ should be practically independent of the amplitude, which is indeed experimentally verified.

For larger amplitudes the active elements do not operate fully linear and the pole positions become signal dependent, resulting in signal dependent damping. The relatively slow amplitude control can not correct this signal dependent damping and Q-tuning current $I_{Q}$ will take on a value which puts the poles just off the $\mathrm{j} \omega$-axis in the RHP for small output signal. In this case $I_{Q}$ does not represent a correct pole position. Furthermore $\mathrm{I}_{\mathrm{Q}}$ will decrease with increasing amplitude.

So to obtain high $\mathrm{Q}_{\text {int }}$ the oscillation amplitude must be chosen in a range where Q-tuning current $\mathrm{I}_{\mathrm{Q}}$ is amplitude independent. Obviously harmonic distortion will be low at such amplitudes.

Compared with the tuning approach based on a voltage controlled filter (VCF) [6], this VCO tuning system has two advantages. First, correct start-up is easier to achieve, because oscillation is the only mode of operation for a VCO while a VCF can either perform as a filter or oscillator, the latter of which must be prevented. Second, the VCO tuning system does not critically depend on the accuracy of amplitude and phase detector, like the VCF system.

For both systems, matching between the integrators in the master VCO or VCF and the slave filter is the ultimate limit for accuracy.

\section{Experimental results}

The tuning circuit was breadboarded for low frequencies using bipolar transistor arrays from a standard $500 \mathrm{MHz}$ technology.

The oscillator was built with $\mathrm{R}_{1}=6.8 \mathrm{k} \Omega$ and $\mathrm{C}_{1}=100 \mathrm{pF}$, resulting in a center frequency of $218 \mathrm{kHz}$ with $V_{C}=0$ Fig. 7 shows $V_{C}$ and $I_{Q}$ versus frequency for $R_{2}=4.64 \mathrm{k} \Omega$, $I_{1}+I_{2}=50 \mu \mathrm{A}, \quad I_{3}=150 \mu \mathrm{A}$ (see fig. 2), the amplifier biased with $I_{S}=125 \mu A, \quad I_{B}=250 \mu \mathrm{A}$ (see fig. 3) and $5 \mathrm{~V}$ total supply voltage. We find $f_{\min }=136 \mathrm{kHz}$ and $f_{\max }=317 \mathrm{kHz}$ which is a tuning range of $-38 \%$ to $+45 \%$ with respect to the center frequency. The pull-in range of the PLL is $136 \mathrm{kHz}$ to $288 \mathrm{kHz}(-38 \%$ to $+32 \%$ tuning), which is sufficient in view of process tolerances and temperature dependence [3].

$$
\text { - vc } \quad-- \text { la }
$$

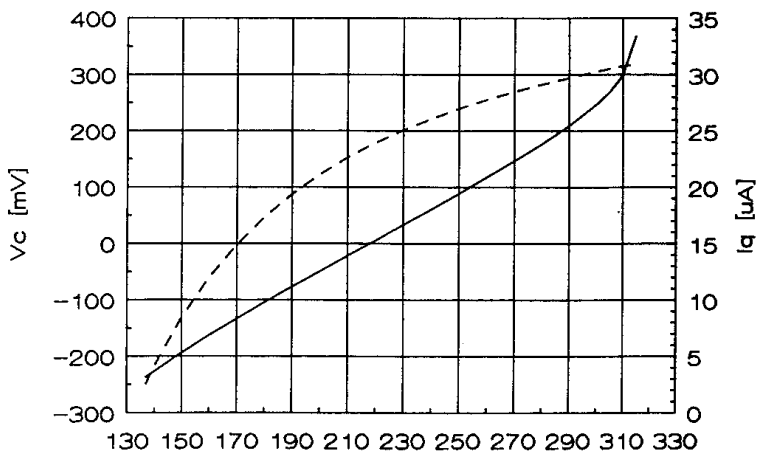

freq. $[\mathrm{kHz}]$

Fig. 7: $V_{C}$ and $I_{Q}$ versus frequency.

$$
f_{\text {OSC }}=140 \mathrm{kHz} \quad f_{O S C}=220 \mathrm{kHz} \quad f_{\text {OSC }}=300 \mathrm{kHz}
$$

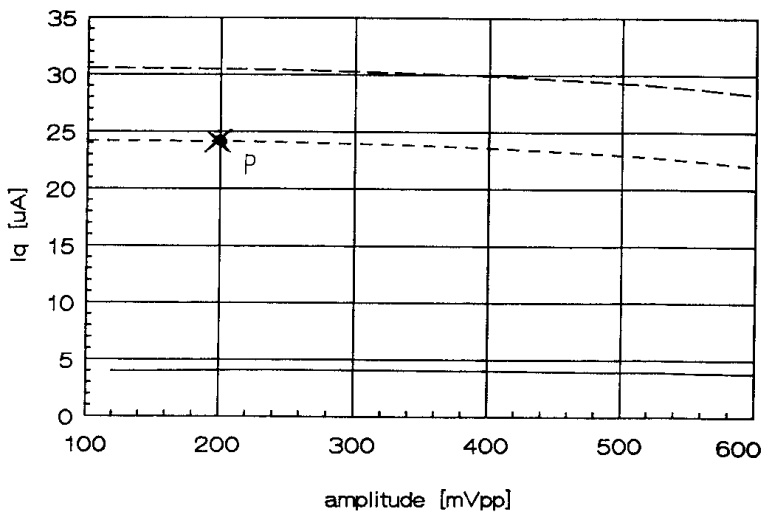

Fig. 8: $\mathrm{I}_{\mathrm{Q}}$ versus oscillation amplitude.

Fig. 8 shows $I_{Q}$ versus amplitude. We see that $I_{Q}$ is practically independent of amplitude. As explained in the previous section this means that a very high $\mathrm{Q}_{\text {int }}$ is realized. At oscillation frequencies of 220 and $300 \mathrm{kHz} \quad \mathrm{I}_{\mathrm{O}}$ slightly decreases for amplitudes above $300 \mathrm{mV}_{\mathrm{pp}}$, because of some damping by non-linearities. Harmonic distortion should be low. Fig. 9 shows the output spectrum of the oscillator at a frequency of $220 \mathrm{kHz}$ and $200 \mathrm{mV}_{\mathrm{pp}}$ amplitude (point $\mathrm{P}$ in fig. 8). The third harmonic is ${ }^{\mathrm{Pp}}-43 \mathrm{~dB}$ down $(0.7 \%$ distortion). This distortion is mainly caused by the PLL. The same measurement with manual frequency control results in only $0.07 \%$ distortion. 


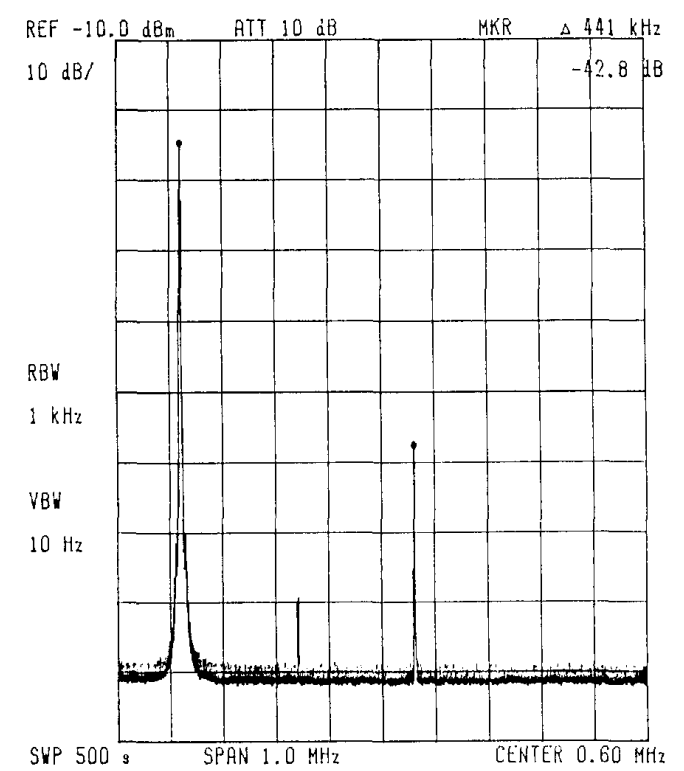

Fig. 9: Oscillator output spectrum.

The oscillator can be changed into a bandpass filter by capacitively coupling an input signal to the input nodes of the first TA in fig. 4, and taking the output signal from the output of the second TA. If the transfer function of this bandpass filter is measured, the oscillator Q-factor can be found from the magnitude response. For this measurement both control loops were opened and $V_{c}$ and $I_{Q}$ were manually set as close as possible to the values for point $\mathrm{P}$ in fig. 8 . The transfer function of fig. 10 was measured with $\mathrm{I}_{\mathrm{O}}$ being only $1 \%$ larger than the closed loop value at point $\mathrm{P}$, in order to damp the oscillator during measurement. Repeated measurements show oscillator Q-factors of 10000 or more. Because $V_{C}$ and $I_{O}$ in this measurement are almost equal to the closed loop values in point $P$, it can be concluded that the tuning circuit yields very high integrator Q-factors.

Fig. 11 shows $I_{Q}$ and the amplitude versus temperature. Reference was a $230 \mathrm{mV}_{\mathrm{pp}}$ square wave, which theoretically would result in a $325 \mathrm{mV}_{\mathrm{pp}}$ oscillation amplitude. The amplitude remains constant within $3 \%$ as temperature increases from $20^{\circ} \mathrm{C}$ to $100^{\circ} \mathrm{C}$. $\mathrm{I}_{\mathrm{O}}$ increases because excess phase increases with temperature due to decreasing $g_{m}$ of the transistors. Correct start-up and convergence of the tuning circuit was verified over this temperature range.

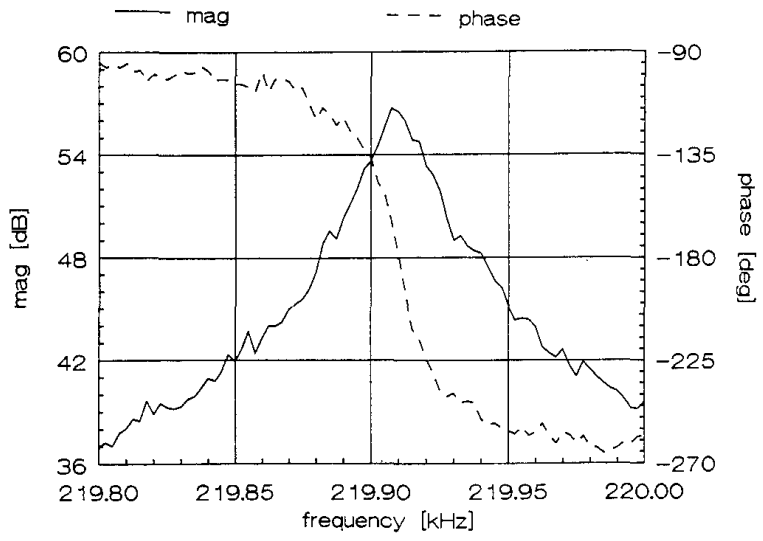

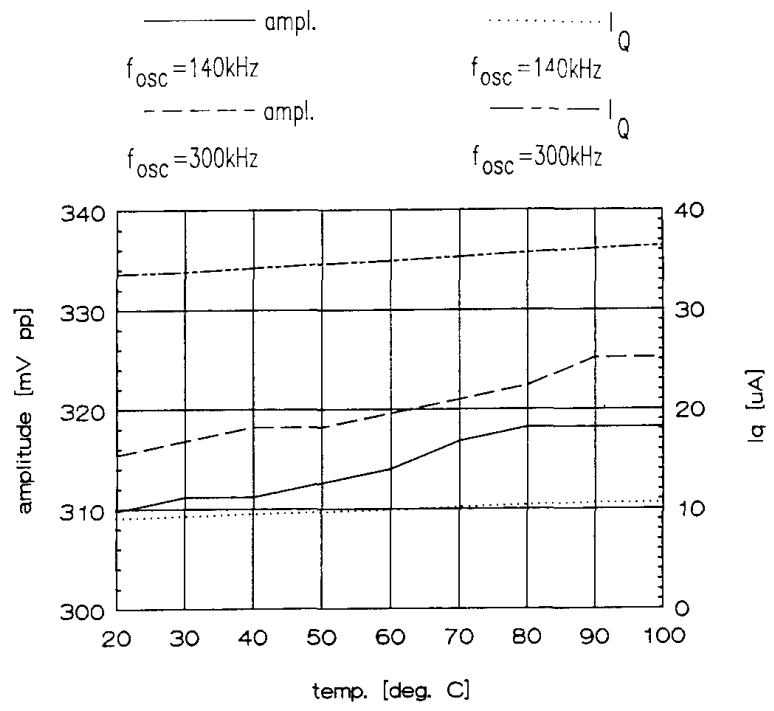

Fig. 11: Oscillation amplitude and $\mathrm{I}_{Q}$ versus temperature.

\section{Conclusions}

In this paper an automatic tuning circuit is presented for tuning both the unity-gain frequency and Q-factor of an integrator circuit for video frequencies [4] using a reference signal of arbitrary shape. Correct start-up and convergence are easily achieved. By means of a low frequency $(136 . .317 \mathrm{kHz})$ breadboard realization it is experimentally verified that very high integrator $\mathrm{Q}$ is achieved for oscillation amplitudes of $300 \mathrm{mV}$ or less. Since Q is controlled via the oscillation amplitude, temperature stability of this amplitude is important. Experimentally the amplitude is found to vary only $3 \%$ for temperatures from $20^{\circ} \mathrm{C}$ up to $100^{\circ} \mathrm{C}$ Correct start-up and convergence were verified over the same temperature range. The circuit will be scaled up in frequency and integrated in a $3 \mathrm{GHz}$ Bi-CMOS process for video filter applications. The amplitude and phase detector have been simulated for video frequencies and correct operation of the integrator at video frequencies has already been verified with a test chip.

The authors wish to thank B. Nauta for fruitful discussions.

\section{References}

[1] S. Takagi, T. Anzai and T. Yanasigawa, "A Differential Input/Output Integrator Without PNP Transistors and its Application to Leapfrog Filter Synthesis", Proc. ISCAS 1988, pp. 2855-2858.

[2] H. Hagiwara et. al., "A Monolithic Video Frequency Filter Using NIC-Based Gyrators", IEEE J. Solid-State Circ., vol..23, pp 175..182, Feb. 1988.

[3] "BIMOS Design Manual", Internal publication of Philips Components, Nijmegen, the Netherlands.

[4] W.J.A. de Heij, E. Seevinck and K. Hoen, "Transconductor and Integrator Circuits for Integrated Bipolar Video Frequency Filters", Proc. ISCAS 1989, pp. 114...117.

[5] Y. Wang, F. Lu and A.A. Abidi, "A $12.5 \mathrm{MHz}$ CMOS Continuous-Time Bandpass Filter", Digest of technical papers, ISSCC 1989.

[6] C.F. Chiou and R. Schaumann,"Design and Performance of a Fully Integrated Bipolar $10.7-\mathrm{MHz}$ Analog Bandpass Filter",IEEE J. Solid-State Circ., vol. SC-21 Feb. 1986 , pp $6 . .14$.

Fig. 10: Oscillator Q-factor measurement. 